\title{
Long-term trends of asthma, allergic rhinitis and atopic eczema in young Finnish men : a retrospective analysis, 1926-2017
}

\section{Reijula, Jere}

2020-12-01

Reijula , J , Latvala , J , Mäkelä , M , Siitonen , S , Saario , M \& Haahtela , T 2020 , ' Long-term trends of asthma, allergic rhinitis and atopic eczema in young Finnish men : a retrospective analysis, 1926-2017' ', European Respiratory Journal , vol. 56 , no. 6 , 1902144 . https://doi.org/10.1183/13993003.02144-2019

http://hdl.handle.net/10138/339897

https://doi.org/10.1183/13993003.02144-2019

unspecified

acceptedVersion

Downloaded from Helda, University of Helsinki institutional repository.

This is an electronic reprint of the original article.

This reprint may differ from the original in pagination and typographic detail.

Please cite the original version. 
ERJ submission 19.3.2020/revised

\section{Long-term trends of asthma, allergic rhinitis and atopic eczema in Finnish young men: a retrospective analysis, 1926-2017}

Jere Reijula, MD, PhD, Department of Pulmonology, Helsinki University Central Hospital, Helsinki University Department of Public Health (jere.reijula@hus.fi)

Jari Latvala, MD, PhD, Finnish Institute of Occupational Health (jari.latvala@ttl.fi)

Mika Mäkelä, MD, PhD, Professor, Department of Dermatology, Allergology and Venereology, Helsinki University Hospital, University of Helsinki (mika.makela@ hus.fi)

Simo Siitonen, MD, PhD, Surgeon General of the Finnish Defence Forces (simo.siitonen@mil.fi)

Mari Saario, Datanome, Project Manager, The Finnish Defence Forces (mari.saario@mil.fi)

Tari Haahtela, MD, PhD, Professor (em.), Department of Dermatology, Allergology and Venereology, Helsinki University Hospital, University of Helsinki (tari.haahtela@ haahtela.fi)

Take-home message: Asthma and allergic conditions have been almost on a linear rise in Finnish young men since the 1960s. The prevalence increase slowed down in the 2000 s and may be levelling off in the 2020s.

Key words: allergic rhinitis, allergy epidemic, asthma, atopic eczema, military service, prevalence

Correspondence: Jere Reijula (jere.reijula@hus.fi) 


\begin{abstract}
Objective: To assess the long-term time trends of the prevalence of asthma, allergic rhinitis and atopic eczema in Finnish young men.
\end{abstract}

Design: A retrospective analysis of cross-sectional data using statistics and reports of Finnish Defence Forces.

Setting: Call-up examinations of candidates for military conscription. Examinations of conscripts discharged from service because of poor health.

Participants: Roughly 1.7 million men, aged $18-19$ years, $98 \%$ of men of conscription age, examined in 1966-2017. A proportional but unknown number of young men examined in 19261961.

Main outcome measures: Asthma recorded at call up examination as the main diagnosis in 19262017 and any diagnosis in 1997-2017. Exemption from military service, and discharge from military service due to asthma. Allergic rhinitis and atopic eczema recorded as the main diagnosis in 1966-2017 and any diagnosis in 1997-2017.

Results: During 1926-61 the prevalence of asthma remained low at between 0.02\% and 0.08\%. A linear rise began between 1961 and 1966, the prevalence increasing from $0.29 \%$ in 1966 to $3.44 \%$ in 2001, a 12-fold rise. Thereafter, prevalence of asthma as the main diagnosis stabilized but continued to increase to $5.19 \%$ in 2017, if also secondary diagnoses of asthma were included. Exemption rates from military service due to asthma have similarly increased but fluctuated more. The prevalence of allergic rhinitis increased from $0.06 \%$ to $10.70 \%$ and atopic eczema from $0.15 \%$ to $2.90 \%$ in $1966-2017$.

Conclusions: In Finland, increase in asthma and allergic conditions among young men became evident in mid 1960s. The increase slowed down in 2000s and may be levelling off in 2020s. 


\section{Introduction}

Asthma and allergy prevalence increase may be levelling off in Europe, although it remains high (1-

4). Globally, the prevalence of asthma is still on rise and people in all world regions are affected (5). In Finland, the recent questionnaire data showed still a slight increase (6) or levelling off (7). We have previously reported asthma and allergy time trends from 1926 up till 2003 in Finnish young men $(8,9)$. We observed a steep rise in the asthma and allergic rhinitis beginning in early 1960s. The prevalence of atopic eczema seemed to reach a plateau already in the 1980s.

Here, we assess the trends of asthma during a 91-year period from 1926 to 2017, and allergic rhinitis as well as atopic eczema from 1966-2017 in young men conscripted for military service in Finland.

\section{Material and Methods}

Roughly $98 \%$ of Finnish men are examined during call up to examine their fitness for compulsory military service. Each conscript's diagnostic codes are registered and held by the Finnish Defence Forces database.

Since 1972 the medical examination has been carried out in two stages. Firstly, the candidate is examined by a general practitioner in a local health center with access to medical records. Fitness is judged on the basis of history, a questionnaire completed by the examinee, and the findings of a clinical examination. When necessary, the candidate is referred to a specialist for further evaluation. $\mathrm{He}$ is then re-examined at call up by the local physician the year prior to the military service. Before 1972, the call up examination was carried out by local physicians appointed by the army.

Medical diagnoses for each person in Finland are made according to the contemporary diagnostic criteria by a licenced physician and are upheld in the patient's medical records. At call up, if the person has multiple diagnoses, the order (primary, secondary, tertiary, quaternary) is determined by the physician according to the eligilibity to serve.

The present cross-sectional, yearly data consist of all Finnish conscripts from 1926 to 1939, 1961 and from 1966 to 2017 . The population comprises of mainly 18 to 19 -year-old Finnish males. No women are included in the data as their service in the military is voluntary. Possible duplicate data, 
i.e. persons who were re-evaluated for military eligibility, are excluded. For "disabling asthma" diagnostic codes are gathered from the exempted person's final follow-up examination, in which the reason for exemption is marked as the primary diagnosis.

The data from the years 1926-1939 and 1961 are obtained from the Finnish Defence Forces annual reports from the Finnish war archives. Since 1966 diagnoses have been registered systematically for each individual and reported annually. Since 1997 also data from secondary, tertiary and quaternary diagnoses have been recorded, in addition to the primary diagnoses. Further details of the call up process have been described earlier (8). During 1966-2017 roughly 1.7 million men were examined.

We included raw pseudonymized data obtained from the Finnish Military registers and collected all diagnoses relating to asthma, allergic rhinitis or atopic eczema in each year's ICD-coding system. Data processing and statistics were made in the Statistics Finland's remote access use (FIONA) - a system employing LibreOffice -software.

\section{Results}

From 1966 to 2017, between 30381 (2016, min) - 36386 (1975, max) men were annually examined in the call up process. The exact number of examined men in 1926-1939 and 1961 was not available but according to the Finnish Defence Forces reports it was in proportion, that is, approximately $98 \%$ of the men in conscription age.

The prevalence of asthma remained low during the first 35-year period (1926-1961). Between 1961-1966 a slight increase was noticed, which accelerated 12-fold during the next 35 years (19662001), from $0.29 \%$ to $3.44 \%$. Since 2001 , asthma as a main (primary) diagnosis remained stable, but the total prevalence, when all diagnoses were included from 1997, showed still increase up till 2012. Thereafter, the prevalence stabilized being 5.19\% in 2017 (Figure 1 A, Suppl. Table 1).

The percentage of men, who prior to the military service, were excluded from the service, because of disabling asthma, increased from 1966 (0.10\%) to 1988 (0.65\%). After that, the exemption rate has fluctuated from $0.17 \%$ to $0.79 \%$. The proportion of men who started the service but were then 
discharged due to asthma exacerbation increased till 2009 after which the rate has decreased slightly, from $1.05 \%$ to $0.87 \%$ by 2017 (Figure 1a).

There was a 100-fold increase in allergic rhinitis as the first diagnosis between 1966-1993, from $0.06 \%$ to $6.46 \%$. The prevalence reached its peak in 2000 (8.88\%). During the last 17 years the prevalence has decreased slightly but remained fairly stable, also when all diagnoses were included. In 2017, the prevalence was $10.70 \%$ (Figure 1 B, Suppl. Table 2).

The prevalence of atopic eczema as a first diagnosis increased 10-fold between 1966-1987, from $0.15 \%$ to $1.47 \%$, reaching then a plateau. However, if all atopic eczema diagnoses were included, a slight upward trend can be seen after 2008, prevalence being 2.90\% in 2017 (Figure 1 C. Suppl. Table 3).

\section{Discussion}

This long-term, nationwide, yearly cross-sectional data comprises of about $98 \%$ of Finnish young men (>30.000 persons annually). Thus the present data offers a unique and robust view of the trends in the asthma and allergy related conditions in Finland.

During 1926-1961 the prevalence of asthma in Finnish young men remained low, but since 1966 increased nearly 12-fold. Partly similar findings have been made in Sweden (10). After 2001, the prevalence of asthma as the main diagnosis seemed to level off, but if also the secondary, tertiary and quaternary diagnoses (recorded only from 1997) were included the rise continued at least up till 2012. This also means that asthma has been noted in the call up examination, but being increasingly asymptomatic or well controlled not regarded as the main diagnosis causing potential disability. The Finnish systematic attempts to improve early detection and treatment may have contributed to this favorable outcome (11).

The same may apply to atopic eczema, which seemed to reach a plateau already in the 1980s. Atopic eczema is often mild enough not causing significant disability, and it may have been increasingly noted at call up as an "add-on-diagnosis". Thus, it has not really levelled off but continued a slow rise. 
Rhinitis and rhinoconjunctivitis are the most common allergic disorders, and prevalence both for main and all diagnoses seems to flatten after in 2000s. This is in agreement with another Finnish study comparing allergic rhinitis in adults from 2006 to 2016 in Helsinki (7).

There has been some variation in the Finnish Defense Forces call up process on the conscripts' eligibility for military service, which may have affected the prevalence of conscripts excluded from military service at call up. However, for long, the policy has been to recruit asthmatic men, if the disease is controlled and the person is motivated for service.

Our findings are in line with the increased allergic manifestations and atopic sensitization, e.g. serum IgE antibodies to pollen allergens, observed in the younger generations in the Finnish but not in Russian Karelia (12). This implies that the increases are largely true and only partly explained by improved awareness and diagnostics. After the second world-war, the Russian Karelian population maintained small-scale agricultural lifestyle while urbanization and affluence took more place on the adjacent Finnish region. The changes in environment and lifestyle, affecting microbial exposure and immune regulation, seem to play a major role in the so called post-war allergy epidemic (13, 14).

We conclude that the increase in asthma and allergy started to become evident in mid 1960s in Finland among young men aged 18-19 years. The increase has started to slow down in 2000s and may be levelling off in 2020s.

Contributors: All authors contributed to conception of the study, and helped to acquire, analyze, and interpret the data and write the article. JR is the guarantor for the paper.

Funding: The Finnish Defence Forces.

Competing interests: None declared.

Ethical approval: Not required. Access to the Finnish Defence Forces databases was granted after a decision of the chief of the army staff on $20^{\text {th }}$ of Dec 2018; Grant nr. AO23452 // 693/12.04.01/2018. 


\section{References}

1. Verlato G, Corsico A, Villani S, et al. Is the prevalence of adult asthma and allergic rhinitis still increasing? Results of an Italian study. J Allergy Clin Immunol 2003;111:1232-8.

2. Braun-Fahrländer C, Gassner M, Grize L, et al. No further increase in asthma, hay fever and atopic sensitisation in adolescence living in Switzerland. Eur Respir J 2004;23: 407-13.

3. Andersson HR, Gupta R, Strachan DP, Limb ES. 50 years of asthma: UK trends from 1955 to 2004. Thorax 2007;62:85-90.

4. Wennergren G, Ekerljung L, Alm B, et al. Asthma in late adolescence-farm childhood is protective and the prevalence increase has levelled off. Pediatr Allergy Immunol 2010; 1:806-13.

5. Anandan C, Nurmatov U, van Schayck OC, Sheikh A. Is the prevalence of asthma declining? Systematic review of epidemiological studies. Allergy 2010;65:152-67.

6. Jousilahti P, Haahtela T, Laatikainen T, et al. Asthma and respiratory allergy prevalence is still increasing among Finnish young adults Eur Respir J 2016;47:985-987.

7. Hisinger-Mölkänen $\mathrm{H}$, Pallasaho $\mathrm{P}$, Haahtela $\mathrm{T}$, et al. The increase of asthma prevalence has levelled off and symptoms decreased in adults during 20 years from 1996 to 2016 in Helsinki, Finland. Respir Med 2019;55:121-126.

8. Haahtela T, Lindholm H, Björkstén F, et al. Prevalence of asthma in Finnish young men. BMJ 1990;301:266-8.

9. Latvala J, von Hertzen L, Lindholm H, Haahtela T. Trends in prevalence of asthma and allergy in Finnish young men: nationwide study, 1966-2003. BMJ 2005; 330:1186-7.

10. Bråbäck L, Hjern A, Rasmussen F. Trends in asthma, allergic rhinitis and eczema among Swedish conscripts from farming and non-farming environments. A nationwide study over three decades. Clin Exp Allergy 2004;34:38-43.

11. Erhola M, Vasankari T, Jormanainen V, et al. 25 years of respiratory health in Finland. Lancet Respir Med 2019;7:e16.

12. Laatikainen T, von Hertzen L, Koskinen JP, et al. Allergy gap between Finnish and Russian Karelia on increase. Allergy 2011; 66:886-92.

13. Hanski I, von Hertzen L, Fyhrquist N, et al. Environmental biodiversity, human microbiota, and allergy are interrelated. Proc Natl Acad Sci U S A 2012;109:8334-9.

14. Haahtela T. A biodiversity hypothesis. Allergy 2019;74:1445-1456. 
Figure 1 A-D. Prevalence of asthma, allergic rhinitis, and atopic eczema in young Finnish men during 1926 (1966) - 2017 at call -up examination for military service. Dotted lines mark all diagnoses (available from 1997) and solid lines main diagnoses. A. Asthma 1926-2017. Prevalence of disabling asthma is shown as percentage of men exempted at call-up (solid gray line) or as percentage of men discharged (of all those who started their service in each year) during service as a result of asthma (dotted gray line). B. Allergic rhinitis 1966-2017. C. Atopic eczema 19662017. D. Asthma, rhinitis and eczema 1966-2017.
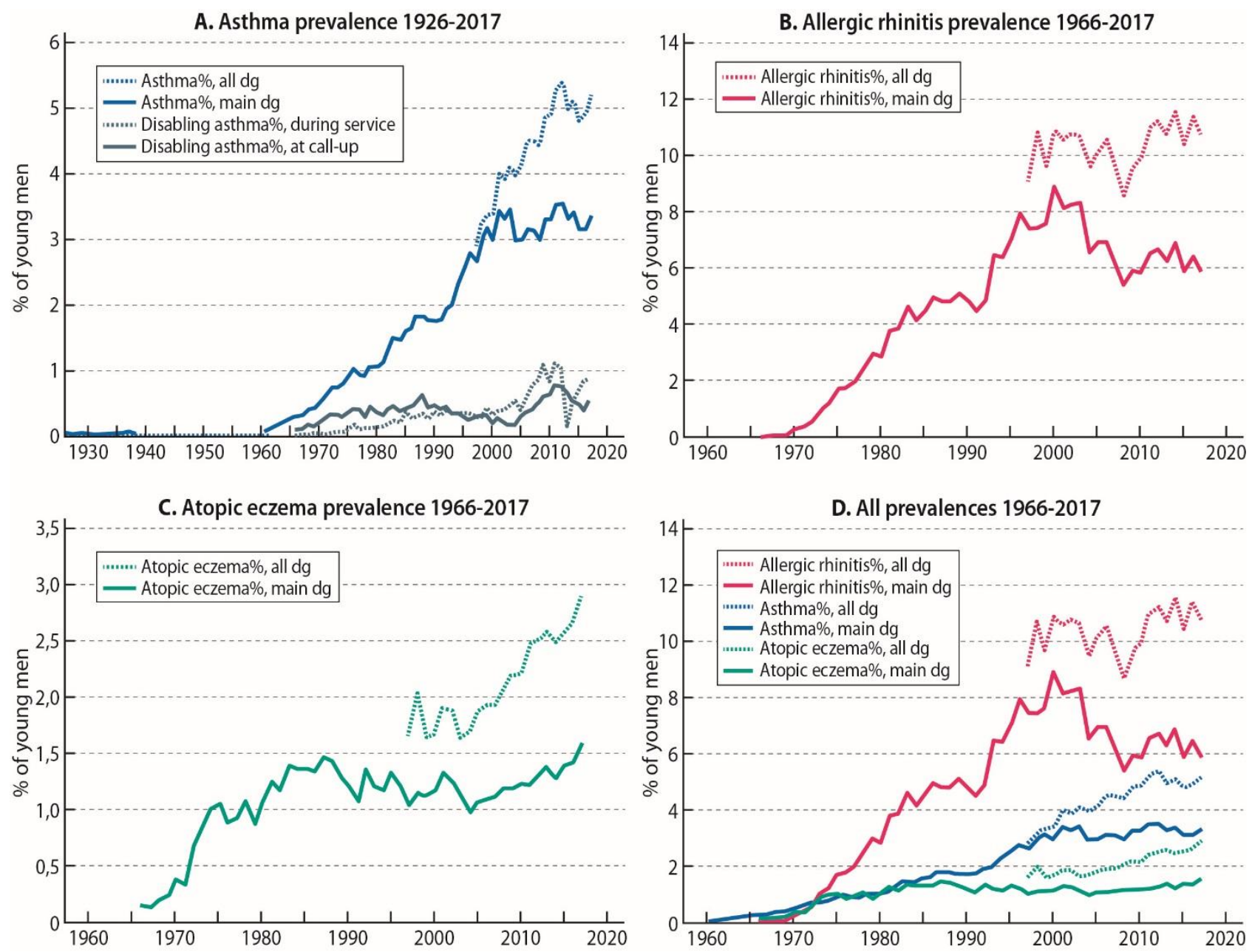
Supplementary Table 1. Number of Finnish conscripts examined each year. Prevalence and 95\% CI of asthma as primary diagnosis from 1966 to 2017, and all diagnoses from 1997 to 2017.

\begin{tabular}{|c|c|c|c|c|}
\hline Year Cohort $n$ & Primary diagnosis \% & $95 \% \mathrm{Cl}$ & All diagnoses \% & $95 \% \mathrm{Cl}$ \\
\hline 1966 & 0,29 & $0,22-0,36$ & & \\
\hline \multicolumn{5}{|l|}{1967} \\
\hline \multicolumn{5}{|l|}{1968} \\
\hline \multicolumn{5}{|l|}{1969} \\
\hline \multicolumn{5}{|l|}{1970} \\
\hline \multicolumn{5}{|l|}{1971} \\
\hline \multicolumn{5}{|l|}{1972} \\
\hline \multicolumn{5}{|l|}{1973} \\
\hline \multicolumn{5}{|l|}{1974} \\
\hline \multirow{2}{*}{\multicolumn{5}{|c|}{$\begin{array}{l}1975 \quad 30300 \\
1976\end{array}$}} \\
\hline & & & & \\
\hline \multicolumn{5}{|l|}{1977} \\
\hline \multicolumn{5}{|l|}{1978} \\
\hline \multicolumn{5}{|l|}{1979} \\
\hline \multicolumn{5}{|l|}{1980} \\
\hline \multicolumn{5}{|l|}{1981} \\
\hline \multicolumn{5}{|l|}{1982} \\
\hline \multicolumn{5}{|l|}{1983} \\
\hline \multicolumn{5}{|l|}{1984} \\
\hline \multicolumn{5}{|l|}{1985} \\
\hline \multicolumn{5}{|l|}{1986} \\
\hline \multicolumn{5}{|l|}{1987} \\
\hline 1988 & & & & \\
\hline 1989 & & & & \\
\hline 1990 & & & & \\
\hline 1991 & & & & \\
\hline 1992 & & & & \\
\hline 1993 & & & & \\
\hline 1994 & & & & \\
\hline 1995 & & & & \\
\hline 1996 & & & & \\
\hline 1997 & 2,65 & $2,48-2,83$ & 2,89 & $2,71-3,07$ \\
\hline 1998 & & & & \\
\hline 1999 & & & & \\
\hline 2000 & & & & \\
\hline 2001 & & & & \\
\hline 2002 & & & & \\
\hline 2003 & & & & \\
\hline 2004 & & & & \\
\hline 2005 & & & & \\
\hline 2006 & & & & \\
\hline 2007 & & & & \\
\hline 2008 & & & & \\
\hline 2009 & & & & \\
\hline 2010 & & & & \\
\hline 2011 & & & & \\
\hline 2012 & & & & \\
\hline 2013 & & & & \\
\hline 2014 & & & & \\
\hline 2015 & & & & \\
\hline $2016 \quad 30381$ & & & & \\
\hline 2017 & & & & \\
\hline
\end{tabular}


Supplementary Table 1. Number of Finnish conscripts examined each year. Prevalence and 95\% CI of allergic rhinitis as primary diagnosis from 1966 to 2017, and all diagnoses from 1997 to 2017.

\begin{tabular}{|c|c|c|c|c|c|}
\hline Year & & Primary diagnosis \% & $95 \% \mathrm{Cl}$ & All diagnoses \% & $95 \% \mathrm{Cl}$ \\
\hline 1966 & & 0,06 & $0,03-0,09$ & & \\
\hline \multicolumn{6}{|l|}{1967} \\
\hline \multicolumn{6}{|l|}{1968} \\
\hline \multicolumn{6}{|l|}{1969} \\
\hline \multicolumn{6}{|l|}{1970} \\
\hline \multicolumn{6}{|l|}{1971} \\
\hline \multicolumn{6}{|l|}{1972} \\
\hline \multicolumn{6}{|l|}{1973} \\
\hline \multicolumn{6}{|l|}{1974} \\
\hline \multicolumn{6}{|c|}{$\begin{array}{ll}1975 & 36386 \\
\end{array}$} \\
\hline \multirow{2}{*}{\multicolumn{6}{|c|}{1976}} \\
\hline \multicolumn{4}{|l|}{1977} & & \\
\hline \multicolumn{6}{|l|}{1978} \\
\hline \multicolumn{6}{|l|}{1979} \\
\hline \multicolumn{6}{|l|}{1980} \\
\hline \multicolumn{6}{|l|}{1981} \\
\hline \multicolumn{6}{|l|}{1982} \\
\hline \multicolumn{6}{|l|}{1983} \\
\hline \multicolumn{6}{|l|}{1984} \\
\hline \multicolumn{6}{|l|}{1985} \\
\hline \multicolumn{6}{|l|}{1986} \\
\hline 1987 & & & & & \\
\hline 1988 & & & & & \\
\hline 1989 & & & & & \\
\hline 1990 & & & & & \\
\hline 1991 & & & & & \\
\hline 1992 & & & & & \\
\hline 1993 & & & & & \\
\hline 1994 & & & & & \\
\hline 1995 & & & & & \\
\hline 1996 & & & & & \\
\hline 1997 & & 7,42 & $7,14-7,70$ & 9,13 & $8,82-9,44$ \\
\hline 1998 & & & & & \\
\hline 1999 & & & & & \\
\hline 2000 & & & & & \\
\hline 2001 & & & & & \\
\hline 2002 & & & & & \\
\hline 2003 & & & & & \\
\hline 2004 & & & & & \\
\hline 2005 & & & & & \\
\hline 2006 & & & & & \\
\hline 2007 & & & & & \\
\hline 2008 & & & & & \\
\hline 2009 & & & & & \\
\hline 2010 & & & & & \\
\hline 2011 & & & & & \\
\hline 2012 & & & & & \\
\hline 2013 & & & & & \\
\hline 2014 & & & & & \\
\hline 2015 & & & & & \\
\hline 2016 & 30381 & & & & \\
\hline 2017 & & & & & \\
\hline
\end{tabular}


Supplementary Table 3. Number of Finnish conscripts examined each year. Prevalence and 95\% CI of atopic eczema as primary diagnosis from 1966 to 2017, and all diagnoses from 1997 to 2017.

\begin{tabular}{|c|c|c|c|c|c|}
\hline Year & & Primary diagnosis \% & $95 \% \mathrm{Cl}$ & All diagnoses \% & $95 \% \mathrm{Cl}$ \\
\hline 1966 & & 0,15 & $0,10-0,20$ & & \\
\hline \multicolumn{6}{|l|}{1967} \\
\hline \multicolumn{6}{|l|}{1968} \\
\hline \multicolumn{6}{|l|}{1969} \\
\hline \multicolumn{6}{|l|}{1970} \\
\hline \multicolumn{6}{|l|}{1971} \\
\hline \multicolumn{6}{|l|}{1972} \\
\hline \multicolumn{6}{|l|}{1973} \\
\hline \multicolumn{6}{|l|}{1974} \\
\hline \multirow{2}{*}{\multicolumn{6}{|c|}{1976}} \\
\hline & & & & & \\
\hline \multicolumn{6}{|l|}{1977} \\
\hline \multicolumn{6}{|l|}{1978} \\
\hline \multicolumn{6}{|l|}{1979} \\
\hline \multicolumn{6}{|l|}{1980} \\
\hline \multicolumn{6}{|l|}{1981} \\
\hline \multicolumn{6}{|l|}{1982} \\
\hline \multicolumn{6}{|l|}{1983} \\
\hline \multicolumn{6}{|l|}{1984} \\
\hline \multicolumn{6}{|l|}{1985} \\
\hline \multicolumn{6}{|l|}{1986} \\
\hline \multicolumn{6}{|l|}{1987} \\
\hline 1988 & & & & & \\
\hline 1989 & & & & & \\
\hline 1990 & & & & & \\
\hline 1991 & & & & & \\
\hline 1992 & & & & & \\
\hline 1993 & & & & & \\
\hline 1994 & & & & & \\
\hline 1995 & & & & & \\
\hline 1996 & & & & & \\
\hline 1997 & & 1,05 & $0,94-1,16$ & 1,67 & $1,53-1,80$ \\
\hline 1998 & & & & & \\
\hline 1999 & & & & & \\
\hline 2000 & & & & & \\
\hline 2001 & & & & & \\
\hline 2002 & & & & & \\
\hline 2003 & & & & & \\
\hline 2004 & & & & & \\
\hline 2005 & & & & & \\
\hline 2006 & & & & & \\
\hline 2007 & & & & & \\
\hline 2008 & & & & & \\
\hline 2009 & & & & & \\
\hline 2010 & & & & & \\
\hline 2011 & & & & & \\
\hline 2012 & & & & & \\
\hline 2013 & & & & & \\
\hline 2014 & & & & & \\
\hline 2015 & & & & & \\
\hline 2016 & 30381 & & & & \\
\hline 2017 & & & & & \\
\hline
\end{tabular}


Table 1. Prevalence and 95\% CI of asthma among Finnish conscripts, primary diagnosis (1.dg) and all diagnoses (all dg)

\begin{tabular}{|c|c|c|c|c|c|c|c|c|}
\hline Year & $\begin{array}{l}\text { 1.dg } \\
(\%)\end{array}$ & & $\begin{array}{r}95 \% \\
\text { CI }\end{array}$ & & $\begin{array}{c}\text { All dg } \\
(\%)\end{array}$ & & $\begin{array}{r}95 \% \\
\text { CI }\end{array}$ & \\
\hline 1966 & 0,29 & 0,22 & - & 0,36 & & & & \\
\hline 1967 & $\mathbf{0 , 3 2}$ & 0,25 & - & 0,39 & & & & \\
\hline 1968 & 0,42 & 0,34 & - & 0,50 & & & & \\
\hline 1969 & 0,44 & 0,36 & - & 0,52 & & & & \\
\hline 1970 & $\mathbf{0 , 5 1}$ & 0,42 & - & 0,60 & & & & \\
\hline 1971 & $\mathbf{0 , 5 8}$ & 0,48 & - & 0,68 & & & & \\
\hline 1972 & 0,74 & 0,63 & - & 0,85 & & & & \\
\hline 1973 & 0,75 & 0,64 & - & 0,86 & & & & \\
\hline 1974 & $\mathbf{0 , 7 9}$ & 0,68 & - & 0,90 & & & & \\
\hline 1975 & $\mathbf{0 , 9 3}$ & 0,83 & - & 1,03 & & & & \\
\hline 1976 & 1,04 & 0,93 & - & 1,15 & & & & \\
\hline 1977 & 0,94 & 0,84 & - & 1,04 & & & & \\
\hline 1978 & $\mathbf{0 , 9 3}$ & 0,83 & - & 1,03 & & & & \\
\hline 1979 & 1,08 & 0,97 & - & 1,19 & & & & \\
\hline 1980 & $\mathbf{1 , 0 7}$ & 0,96 & - & 1,18 & & & & \\
\hline 1981 & 1,14 & 1,03 & - & 1,25 & & & & \\
\hline 1982 & 1,25 & 1,13 & - & 1,37 & & & & \\
\hline 1983 & 1,52 & 1,39 & - & 1,65 & & & & \\
\hline 1984 & 1,48 & 1,35 & - & 1,61 & & & & \\
\hline 1985 & 1,61 & 1,47 & - & 1,75 & & & & \\
\hline 1986 & 1,65 & 1,51 & - & 1,79 & & & & \\
\hline 1987 & 1,84 & 1,69 & - & 1,99 & & & & \\
\hline 1988 & $\mathbf{1 , 8 7}$ & 1,71 & - & 2,03 & & & & \\
\hline 1989 & 1,79 & 1,64 & - & 1,94 & & & & \\
\hline 1990 & 1,77 & 1,63 & - & 1,90 & & & & \\
\hline 1991 & 1,78 & 1,64 & - & 1,92 & & & & \\
\hline 1992 & 1,95 & 1,80 & - & 2,10 & & & & \\
\hline 1993 & 2,02 & 1,87 & - & 2,17 & & & & \\
\hline 1994 & 2,37 & 2,21 & - & 2,53 & & & & \\
\hline 1995 & 2,54 & 2,37 & - & 2,71 & & & & \\
\hline 1996 & 2,79 & 2,61 & - & 2,97 & & & & \\
\hline 1997 & 2,65 & 2,48 & - & 2,83 & 2,89 & 2,71 & - & 3,07 \\
\hline 1998 & 2,98 & 2,80 & - & 3,17 & 3,24 & 3,05 & - & 3,43 \\
\hline 1999 & 3,18 & 2,99 & - & 3,36 & $\mathbf{3 , 3 7}$ & 3,18 & - & 3,57 \\
\hline 2000 & 2,98 & 2,80 & - & 3,16 & $\mathbf{3 , 3 9}$ & 3,20 & - & 3,58 \\
\hline 2001 & 3,44 & 3,25 & - & 3,63 & 4,01 & 3,81 & - & 4,22 \\
\hline 2002 & 3,31 & 3,12 & - & 3,50 & 3,90 & 3,70 & - & 4,11 \\
\hline 2003 & 3,45 & 3,25 & - & 3,64 & 4,10 & 3,89 & - & 4,31 \\
\hline 2004 & 2,98 & 2,79 & - & 3,16 & 3,95 & 3,74 & - & 4,17 \\
\hline
\end{tabular}




\begin{tabular}{l|llllllll}
$\mathbf{2 0 0 5}$ & $\mathbf{2 , 9 9}$ & 2,81 & - & 3,18 & $\mathbf{4 , 1 4}$ & 3,92 & - & 4,36 \\
$\mathbf{2 0 0 6}$ & $\mathbf{3 , 1 5}$ & 2,96 & - & 3,34 & $\mathbf{4 , 4 9}$ & 4,27 & - & 4,71 \\
$\mathbf{2 0 0 7}$ & $\mathbf{3 , 1 2}$ & 2,94 & - & 3,31 & $\mathbf{4 , 5 1}$ & 4,29 & - & 4,73 \\
$\mathbf{2 0 0 8}$ & $\mathbf{3 , 0 0}$ & 2,82 & - & 3,18 & $\mathbf{4 , 4 1}$ & 4,20 & - & 4,63 \\
$\mathbf{2 0 0 9}$ & $\mathbf{3 , 3 1}$ & 3,12 & - & 3,49 & $\mathbf{4 , 8 6}$ & 4,63 & - & 5,09 \\
$\mathbf{2 0 1 0}$ & $\mathbf{3 , 3 1}$ & 3,12 & - & 3,49 & $\mathbf{4 , 8 6}$ & 4,63 & - & 5,08 \\
$\mathbf{2 0 1 1}$ & $\mathbf{3 , 5 2}$ & 3,33 & - & 3,72 & $\mathbf{5 , 2 7}$ & 5,04 & - & 5,51 \\
$\mathbf{2 0 1 2}$ & $\mathbf{3 , 5 3}$ & 3,33 & - & 3,72 & $\mathbf{5 , 3 8}$ & 5,14 & - & 5,62 \\
$\mathbf{2 0 1 3}$ & $\mathbf{3 , 3 1}$ & 3,12 & - & 3,50 & $\mathbf{4 , 9 6}$ & 4,73 & - & 5,19 \\
$\mathbf{2 0 1 4}$ & $\mathbf{3 , 4 0}$ & 3,20 & - & 3,60 & $\mathbf{5 , 1 0}$ & 4,86 & - & 5,34 \\
$\mathbf{2 0 1 5}$ & $\mathbf{3 , 1 6}$ & 2,97 & - & 3,35 & $\mathbf{4 , 8 1}$ & 4,57 & - & 5,05 \\
$\mathbf{2 0 1 6}$ & $\mathbf{3 , 1 6}$ & 2,96 & - & 3,36 & $\mathbf{4 , 8 9}$ & 4,65 & - & 5,14 \\
$\mathbf{2 0 1 7}$ & $\mathbf{3 , 3 4}$ & 3,13 & - & 3,54 & $\mathbf{5 , 1 9}$ & 4,94 & - & 5,44
\end{tabular}


Table 2. Prevalence and 95\% CI of allergic rhinitis among Finnish conscripts, primary diagnosis (1.dg) and all diagnoses (all dg)

\begin{tabular}{|c|c|c|c|c|c|c|c|c|}
\hline \multirow{2}{*}{$\frac{\text { Year }}{1966}$} & $\begin{array}{l}\text { 1.dg } \\
(\%)\end{array}$ & \multicolumn{3}{|c|}{$\begin{array}{c}95 \% \\
\text { CI }\end{array}$} & All dg (\%) & \multicolumn{3}{|c|}{$95 \%$} \\
\hline & 0,06 & 0,03 & - & 0,09 & & & & \\
\hline 1967 & 0,06 & 0,03 & - & 0,09 & & & & \\
\hline 1968 & $\mathbf{0 , 0 7}$ & 0,04 & - & 0,10 & & & & \\
\hline 1969 & 0,09 & 0,05 & - & 0,13 & & & & \\
\hline 1970 & $\mathbf{0 , 3 1}$ & 0,24 & - & 0,38 & & & & \\
\hline 1971 & $\mathbf{0 , 4 3}$ & 0,35 & - & 0,51 & & & & \\
\hline 1972 & 0,62 & 0,52 & - & 0,72 & & & & \\
\hline 1973 & 1,00 & 0,87 & - & 1,13 & & & & \\
\hline 1974 & 1,28 & 1,14 & - & 1,42 & & & & \\
\hline 1975 & 1,73 & 1,60 & - & 1,86 & & & & \\
\hline 1976 & $\mathbf{1 , 8 0}$ & 1,66 & - & 1,94 & & & & \\
\hline 1977 & 2,04 & 1,89 & - & 2,19 & & & & \\
\hline 1978 & 2,54 & 2,37 & - & 2,71 & & & & \\
\hline 1979 & 2,98 & 2,80 & - & 3,16 & & & & \\
\hline 1980 & 2,88 & 2,71 & - & 3,05 & & & & \\
\hline 1981 & 3,81 & 3,61 & - & 4,01 & & & & \\
\hline 1982 & 3,90 & 3,69 & - & 4,11 & & & & \\
\hline 1983 & 4,66 & 4,43 & - & 4,89 & & & & \\
\hline 1984 & 4,16 & 3,94 & - & 4,38 & & & & \\
\hline 1985 & 4,53 & 4,30 & - & 4,76 & & & & \\
\hline 1986 & 4,99 & 4,75 & - & 5,23 & & & & \\
\hline 1987 & 4,82 & 4,58 & - & 5,06 & & & & \\
\hline 1988 & 4,82 & 4,57 & - & 5,07 & & & & \\
\hline 1989 & 5,11 & 4,86 & - & 5,36 & & & & \\
\hline 1990 & 4,82 & 4,60 & - & 5,04 & & & & \\
\hline 1991 & 4,51 & 4,29 & - & 4,74 & & & & \\
\hline 1992 & 4,90 & 4,67 & - & 5,13 & & & & \\
\hline 1993 & 6,46 & 6,20 & - & 6,72 & & & & \\
\hline 1994 & 6,40 & 6,14 & - & 6,65 & & & & \\
\hline 1995 & 7,04 & 6,76 & - & 7,32 & & & & \\
\hline 1996 & 7,94 & 7,65 & - & 8,23 & & & & \\
\hline 1997 & 7,42 & 7,14 & - & 7,70 & 9,13 & 8,82 & - & 9,44 \\
\hline 1998 & 7,43 & 7,15 & - & 7,72 & 10,77 & 10,44 & - & 11,11 \\
\hline 1999 & 7,60 & 7,32 & - & 7,88 & 9,64 & 9,32 & - & 9,96 \\
\hline 2000 & $\mathbf{8 , 8 8}$ & 8,58 & - & 9,18 & 10,84 & 10,52 & - & 11,17 \\
\hline 2001 & 8,14 & 7,85 & - & 8,42 & 10,53 & 10,21 & - & 10,85 \\
\hline 2002 & 8,25 & 7,96 & - & 8,54 & 10,77 & 10,44 & - & 11,09 \\
\hline 2003 & 8,33 & 8,03 & - & 8,63 & 10,64 & 10,31 & - & 10,97 \\
\hline 2004 & 6,56 & 6,29 & - & 6,83 & 9,52 & 9,20 & - & 9,84 \\
\hline
\end{tabular}




\begin{tabular}{l|cccccccc}
$\mathbf{2 0 0 5}$ & $\mathbf{6 , 9 3}$ & 6,65 & - & 7,21 & $\mathbf{1 0 , 1 7}$ & 9,83 & - & 10,50 \\
$\mathbf{2 0 0 6}$ & $\mathbf{6 , 9 4}$ & 6,67 & - & 7,21 & $\mathbf{1 0 , 5 5}$ & 10,22 & - & 10,88 \\
$\mathbf{2 0 0 7}$ & $\mathbf{6 , 2 2}$ & 5,96 & - & 6,48 & $\mathbf{9 , 6 6}$ & 9,34 & - & 9,97 \\
$\mathbf{2 0 0 8}$ & $\mathbf{5 , 4 0}$ & 5,17 & - & 5,64 & $\mathbf{8 , 5 7}$ & 8,28 & - & 8,87 \\
$\mathbf{2 0 0 9}$ & $\mathbf{5 , 9 2}$ & 5,68 & - & 6,17 & $\mathbf{9 , 5 6}$ & 9,25 & - & 9,87 \\
$\mathbf{2 0 1 0}$ & $\mathbf{5 , 8 7}$ & 5,63 & - & 6,12 & $\mathbf{9 , 9 1}$ & 9,60 & - & 10,22 \\
$\mathbf{2 0 1 1}$ & $\mathbf{6 , 5 6}$ & 6,30 & - & 6,83 & $\mathbf{1 0 , 9 7}$ & 10,64 & - & 11,30 \\
$\mathbf{2 0 1 2}$ & $\mathbf{6 , 7 1}$ & 6,45 & - & 6,97 & $\mathbf{1 1 , 2 1}$ & 10,88 & - & 11,55 \\
$\mathbf{2 0 1 3}$ & $\mathbf{6 , 2 7}$ & 6,01 & - & 6,53 & $\mathbf{1 0 , 7 3}$ & 10,39 & - & 11,06 \\
$\mathbf{2 0 1 4}$ & $\mathbf{6 , 8 6}$ & 6,58 & - & 7,13 & $\mathbf{1 1 , 5 3}$ & 11,18 & - & 11,88 \\
$\mathbf{2 0 1 5}$ & $\mathbf{5 , 8 9}$ & 5,63 & - & 6,15 & $\mathbf{1 0 , 3 7}$ & 10,03 & - & 10,70 \\
$\mathbf{2 0 1 6}$ & $\mathbf{6 , 4 6}$ & 6,18 & - & 6,73 & $\mathbf{1 1 , 3 8}$ & 11,02 & - & 11,74 \\
$\mathbf{2 0 1 7}$ & $\mathbf{5 , 9 0}$ & 5,63 & - & 6,16 & $\mathbf{1 0 , 7 0}$ & 10,35 & - & 11,04
\end{tabular}


Table 3. Prevalence and 95\% CI of atopic ezcema among

Finnish conscripts, primary diagnosis (1.dg) and all diagnoses (all dg)

\begin{tabular}{|c|c|c|c|c|c|c|c|c|}
\hline \multirow{2}{*}{$\frac{\text { Year }}{1966}$} & $\begin{array}{l}1 . d g \\
(\%)\end{array}$ & & $\begin{array}{r}95 \% \\
\text { CI }\end{array}$ & & \multirow[t]{2}{*}{$\begin{array}{c}\text { All } \\
\mathbf{d g} \\
(\%)\end{array}$} & \multicolumn{3}{|c|}{$\begin{array}{c}95 \% \\
\text { CI } \\
\end{array}$} \\
\hline & 0,15 & 0,10 & - & 0,20 & & & & \\
\hline 1967 & 0,14 & 0,09 & - & 0,19 & & & & \\
\hline 1968 & 0,20 & 0,14 & - & 0,26 & & & & \\
\hline 1969 & 0,25 & 0,19 & - & 0,31 & & & & \\
\hline 1970 & 0,39 & 0,31 & - & 0,47 & & & & \\
\hline 1971 & 0,34 & 0,27 & - & 0,41 & & & & \\
\hline 1972 & 0,68 & 0,58 & - & 0,78 & & & & \\
\hline 1973 & 0,86 & 0,74 & - & 0,98 & & & & \\
\hline 1974 & 1,01 & 0,88 & - & 1,14 & & & & \\
\hline 1975 & 1,05 & 0,95 & - & 1,15 & & & & \\
\hline 1976 & $0, \mathbf{8 8}$ & 0,78 & - & 0,98 & & & & \\
\hline 1977 & 0,94 & 0,84 & - & 1,04 & & & & \\
\hline 1978 & 1,08 & 0,97 & - & 1,19 & & & & \\
\hline 1979 & 0,86 & 0,76 & - & 0,96 & & & & \\
\hline 1980 & 1,07 & 0,96 & - & 1,18 & & & & \\
\hline 1981 & 1,26 & 1,14 & - & 1,38 & & & & \\
\hline 1982 & 1,18 & 1,07 & - & 1,29 & & & & \\
\hline 1983 & 1,40 & 1,27 & - & 1,53 & & & & \\
\hline 1984 & 1,36 & 1,24 & - & 1,48 & & & & \\
\hline 1985 & 1,37 & 1,24 & - & 1,50 & & & & \\
\hline 1986 & 1,34 & 1,22 & - & 1,46 & & & & \\
\hline 1987 & 1,47 & 1,34 & - & 1,60 & & & & \\
\hline 1988 & 1,43 & 1,29 & - & 1,57 & & & & \\
\hline 1989 & 1,29 & 1,16 & - & 1,42 & & & & \\
\hline 1990 & 1,21 & 1,10 & - & 1,32 & & & & \\
\hline 1991 & 1,08 & 0,97 & - & 1,19 & & & & \\
\hline 1992 & 1,36 & 1,24 & - & 1,48 & & & & \\
\hline 1993 & 1,21 & 1,09 & - & 1,33 & & & & \\
\hline 1994 & 1,17 & 1,06 & - & 1,28 & & & & \\
\hline 1995 & 1,33 & 1,21 & - & 1,45 & & & & \\
\hline 1996 & 1,21 & 1,09 & - & 1,33 & & & & \\
\hline 1997 & 1,05 & 0,94 & - & 1,16 & 1,67 & 1,53 & - & 1,80 \\
\hline 1998 & 1,15 & 1,04 & - & 1,26 & 2,03 & 1,88 & - & 2,18 \\
\hline 1999 & 1,12 & 1,01 & - & 1,23 & 1,62 & 1,49 & - & 1,76 \\
\hline 2000 & 1,17 & 1,06 & - & 1,28 & 1,70 & 1,57 & - & 1,84 \\
\hline 2001 & 1,33 & 1,21 & - & 1,45 & 1,91 & 1,76 & - & 2,05 \\
\hline 2002 & 1,25 & 1,13 & - & 1,37 & 1,88 & 1,73 & - & 2,02 \\
\hline 2003 & 1,13 & 1,02 & - & 1,24 & 1,63 & 1,50 & - & 1,77 \\
\hline
\end{tabular}




\begin{tabular}{l|llllllll}
$\mathbf{2 0 0 4}$ & $\mathbf{0 , 9 8}$ & 0,87 & - & 1,09 & $\mathbf{1 , 7 0}$ & 1,56 & - & 1,84 \\
$\mathbf{2 0 0 5}$ & $\mathbf{1 , 0 7}$ & 0,96 & - & 1,18 & $\mathbf{1 , 8 6}$ & 1,71 & - & 2,01 \\
$\mathbf{2 0 0 6}$ & $\mathbf{1 , 1 1}$ & 1,00 & - & 1,22 & $\mathbf{1 , 9 4}$ & 1,79 & - & 2,09 \\
$\mathbf{2 0 0 7}$ & $\mathbf{1 , 1 1}$ & 1,00 & - & 1,22 & $\mathbf{1 , 9 3}$ & 1,79 & - & 2,08 \\
$\mathbf{2 0 0 8}$ & $\mathbf{1 , 1 9}$ & 1,08 & - & 1,30 & $\mathbf{2 , 0 9}$ & 1,94 & - & 2,24 \\
$\mathbf{2 0 0 9}$ & $\mathbf{1 , 2 0}$ & 1,09 & - & 1,31 & $\mathbf{2 , 2 0}$ & 2,04 & - & 2,35 \\
$\mathbf{2 0 1 0}$ & $\mathbf{1 , 2 4}$ & 1,12 & - & 1,36 & $\mathbf{2 , 2 1}$ & 2,06 & - & 2,36 \\
$\mathbf{2 0 1 1}$ & $\mathbf{1 , 2 2}$ & 1,10 & - & 1,34 & $\mathbf{2 , 4 8}$ & 2,31 & - & 2,64 \\
$\mathbf{2 0 1 2}$ & $\mathbf{1 , 3 0}$ & 1,18 & - & 1,42 & $\mathbf{2 , 5 0}$ & 2,33 & - & 2,66 \\
$\mathbf{2 0 1 3}$ & $\mathbf{1 , 3 8}$ & 1,25 & - & 1,51 & $\mathbf{2 , 5 8}$ & 2,41 & - & 2,75 \\
$\mathbf{2 0 1 4}$ & $\mathbf{1 , 2 8}$ & 1,16 & - & 1,40 & $\mathbf{2 , 4 7}$ & 2,30 & - & 2,64 \\
$\mathbf{2 0 1 5}$ & $\mathbf{1 , 3 9}$ & 1,26 & - & 1,52 & $\mathbf{2 , 5 5}$ & 2,38 & - & 2,72 \\
$\mathbf{2 0 1 6}$ & $\mathbf{1 , 4 2}$ & 1,29 & - & 1,55 & $\mathbf{2 , 6 5}$ & 2,47 & - & 2,83 \\
$\mathbf{2 0 1 7}$ & $\mathbf{1 , 5 9}$ & 1,45 & - & 1,73 & $\mathbf{2 , 9 0}$ & 2,71 & - & 3,09
\end{tabular}

